\title{
Hypermutation induced by APOBEC-1 overexpression can be eliminated
}

\author{
ZHIGANG CHEN, ${ }^{1}$ THOMAS L. EGGERMAN, ${ }^{1,2}$ ALEXANDER V. BOCHAROV ${ }^{1}$ IRINA N. BARANOVA, ${ }^{1}$ \\ TATYANA G. VISHNYAKOVA, ${ }^{1}$ GYORGY CSAKO, ${ }^{1}$ and AMY P. PATTERSON ${ }^{1,3}$ \\ ${ }^{1}$ Department of Laboratory Medicine, Clinical Center, National Institutes of Health, Bethesda, Maryland 20892, USA \\ ${ }^{2}$ Division of Diabetes, Endocrinology and Metabolic Diseases, National Institute of Diabetes and Digestive and Kidney Diseases, \\ National Institutes of Health, Bethesda, Maryland 20892, USA \\ ${ }^{3}$ Office of Science Policy, Office of the Director, National Institutes of Health, Bethesda, Maryland 20892, USA
}

\begin{abstract}
APOBEC-1 overexpression in liver has been shown to effectively reduce apoB-100 levels. However, nonspecific hypermutation and liver tumor formation potentially related to hypermutation in transgenic animals compromise its potential use for gene therapy. In studying apoB mRNA editing regulation, we found that the core editing auxiliary factor ACF dose-dependently increases APOBEC-1 nonspecific hypermutation and specific editing with variable site sensitivity. Overexpression of APOBEC-1 together with ACF in human hepatic HepG2 cells hypermutated apoB mRNAs 20\%-65\% at sites 6639, 6648, 6655, 6762, 6802, and 6845, in addition to the normal $90 \%$ editing at 6666 . The hypermutation activity of APOBEC-1 was decreased to background levels by a single point APOBEC-1 mutation of P29F or E181Q, while $50 \%$ of wild-type control editing at the normal site was retained. The hypermutations on both apoB and novel APOBEC-1 target 1 (NAT1) mRNA were also decreased to background levels with P29F and E181Q mutants in rat liver primary culture cells. The loss of hypermutation with the mutants was associated with significantly decreased APOBEC-1/ACF interaction. These data suggest that nonspecific hypermutation induced by overexpressing APOBEC-1 can be virtually eliminated by site-specific mutation, while maintaining specific editing activity at the normal site, reopening the potential use of APOBEC-1 gene therapy for hyperlipidemia.
\end{abstract}

Keywords: apolipoprotein; editing; hyperediting; APOBEC-1; ACF; hypermutation

\section{INTRODUCTION}

Apolipoproteins are carrier proteins for lipids, facilitating lipid transport and exchange. Apolipoprotein B (apoB) is an integral and nonexchangeable structural component of lipoprotein particles, including chylomicrons, very-low-density lipoprotein (VLDL), and low-density lipoprotein (LDL), playing a central role in lipoprotein metabolism (Young 1990). ApoB circulates in human plasma as two isoforms, apoB-100 and apoB-48. ApoB-48 is generated by apoB-100 mRNA editing through a catalytic component APOBEC-1 and auxiliary cofactors that convert codon 2153 (CAA) to a translation stop codon (UAA) (Chen et al. 1987; Powell et al. 1987).

Abbreviations: apo, apolipoprotein; VLDL, very-low-density lipoprotein; LDL, low-density lipoprotein; Lp(a), lipoprotein(a); pe, primer extension.

Reprint requests to: Amy P. Patterson, Office of Science Policy, Office of the Director, National Institutes of Health, 6705 Rockledge Drive, Suite 750, Bethesda, MD 20892, USA; e-mail: pattersa@od.nih.gov; fax: (301) 496-9839.

Article published online ahead of print. Article and publication date are at http://www.rnajournal.org/cgi/doi/10.1261/rna.1863010.
ApoB-48 is synthesized by the intestine in humans and is essential for the assembly of chylomicrons. ApoB-100 is produced in human liver and is required for the synthesis and secretion of VLDL that is metabolized into LDL. LDL transports two-thirds of the plasma cholesterol. An elevated LDL cholesterol level is a major risk factor for developing atherosclerosis (Young 1990; Innerarity et al. 1996). ApoB-100 is virtually the only protein component of LDL.

ApoB mRNA editing occurs predominently in human small intestine, but not in liver, because of tissue specific APOBEC-1 expression. In contrast, rats and mice express APOBEC-1 in multiple tissues including the liver (Innerarity et al. 1996). As a result, rats and mice secrete both apoB-100and apoB-48-containing lipoproteins from the liver, have lower apoB-100 and LDL levels, and are more resistant to diet-induced atherosclerosis than most other species (Young 1990; Chan 1992). Lipoprotein particles containing apoB-48 have multiple copies of apoE, facilitating their clearance through both the LDL receptor and LDL receptor-related protein (Pitas et al. 1980). Consequently, apoB-48 containing particles are cleared from the plasma more rapidly than 
apoB-100 containing LDL (minutes versus 2-3 d) (Innerarity and Mahley 1978).

Hepatic expression of APOBEC-1 has been investigated in animal models to reduce the levels of atherogenic lipoproteins, LDL, and lipoprotein(a) $[\operatorname{Lp}(\mathrm{a})]$. Several reports demonstrated that adenoviral delivery of rat APOBEC-1 to the liver of rabbits and mice or liver-specific overexpression of APOBEC-1 in apoB transgenic mice effectively decreased LDL cholesterol levels (Teng et al. 1994; Yamanaka et al. 1995; Hughes et al. 1996; Kozarsky et al. 1996; Teng et al. 1997; Qian et al. 1998). However, high constitutive APOBEC-1 expression in mouse liver resulted in liver dysplasia and hepatocellular carcinomas (Yamanaka et al. 1995). Analyses of apoB mRNA revealed extensively edited cytidines at multiple sites within the downstream region of the mooring sequence motif and hypermutation was also found over the entire coding sequence of novel APOBEC-1 target 1 (NAT1) (Yamanaka et al. 1996, 1997). Even transient APOBEC-1 expression controlled by a regulatable tet-Off system also leads to significant aberrant hypermutation in mouse liver (Hersberger et al. 2003). The risk of adverse effects stemming from elevated levels of APOBEC-1 expression or uncontrolled APOBEC-1 activity has eliminated its interest as a gene therapy for hyperlipidemia.

ApoB mRNA editing is a precise and efficient deamination reaction under physiological conditions, occurring only on the cytidine at site 6666. Aberrant nonspecific C-to-U transitions were not found even when up to $90 \%$ editing levels were achieved in the small intestine during development or in the mouse liver after fasting and refeeding (Funahashi et al. 1995; Chen et al. 2007). Aberrant hypermutation occurred only when APOBEC-1 was overexpressed, occurring even in cultured cells (Sowden et al. 1996a,b; Siddiqui et al. 1999). Investigation in rat liver McA7777 cells showed that species source of APOBEC-1, sequences of apoB mRNA, and expression of auxiliary proteins could influence editing fidelity (Sowden et al. 1998). The apoB mRNA editing reaction involves the catalytic component APOBEC-1 and auxiliary cofactors, potentially including ACF, CUGBP2, GRY-RBP, hnRNP-C1, ABBP1, ABBP2, $\mathrm{KSRP}$, and BAG4, identified by their interactions with APOBEC-1 or apoB mRNA (Anant et al. 2003). APOBEC-1 and ACF form a minimal editing active complex in vitro. However, the means to overcome hypermutation due to APOBEC-1 overexpression remain elusive. In this article, we used hypermutation induced by adenoviral APOBEC-1 overexpression in HepG2 cells to study the interaction between APOBEC-1 and its editing auxiliary factors and the structural contribution of APOBEC-1 to hypermutation. We found that the core editing cofactor ACF increased APOBEC-1-induced hypermutation. Two single site-directed APOBEC-1 mutations virtually eliminated APOBEC-1 hypermutation, while retaining about half-normal editing activity, reopening the possible use of APOBEC-1 gene therapy for hyperlipidemia.

\section{RESULTS}

\section{Core editing cofactor ACF increases APOBEC-1 hypermutation}

ApoB mRNA editing is exquisitely precise under normal conditions, such as during mouse small intestine development where apoB mRNA editing increases from $\sim 3 \%$ to $\sim 90 \%$ without any hypermutation. The editing increase begins with decreased levels of inhibitory CUGBP2 expression followed by increased levels of APOBEC-1 and ACF (four- and eightfold) and then by decreased levels of the inhibitory components GRY-RBP and hnRNP-C1 (75\% and 56\%) (Chen et al. 2007), indicating that cooperatively expressed cofactors may play an important role in the regulation of editing magnitude and specificity. To search for a potential approach to minimize hypermutation, we first analyzed the contribution of potential cofactors to hypermutation by coexpressing adenoviral APOBEC-1 with each individual cofactor in HepG2 cells, including ACF, CUGBP2, GRY-RBP, KSRP, hnRNP-C1, ABBP1, ABBP2, and BAG4. HepG2 cells have no apoB mRNA editing due to absent APOBEC-1 expression. Overexpression of APOBEC-1 alone resulted in $69.5 \%$ editing of apoB mRNA at site 6666 and $8.0 \%$ hypermutation at site 6655 . Coexpression of APOBEC-1 and ACF increased normal editing from $69.6 \%$ to $87.9 \%$ and hypermutation from $8.0 \%$ to $31.6 \%$ (Fig. $1 \mathrm{I}, \mathrm{G})$, while the other cofactors resulted in small or insignificant changes (data not shown). These data suggest that hypermutation induced by APOBEC-1 overexpression cannot be eliminated by coexpression of potential editing cofactors and the major core cofactor ACF plays an increased role on APOBEC-1 editing and hypermutation.

To obtain detailed specific information on apoB mRNA hypermutation induced by APOBEC-1 overexpression in HepG2 cells, we investigated hypermutation sites and mutation rates by clonal sequencing. Adenoviral noncoding gene vector control, APOBEC-1, and APOBEC-1 plus ACF were transiently overexpressed in HepG2 cells. ApoB mRNA from 6471 to 6886 covering the editing area was PCR-amplified, cloned into a pENTR1A vector, and sequenced by randomly selected 20 clones. As summarized in Figure 1A, C-to-U transitions occurred at multiple sites including 6623, 6639, 6645, 6648, 6651, 6666, 6762, 6802, and 6845. These C-to-U transitions resulted in protein translation stop codons for sites 6645, 6651, and 6666 or coding mutations for sites 6655 (Thr/Ile), 6762 (Leu/Phe), and 6802 (Thr/Ile). The C-to-U transitions at sites 6623, 6639,6648 , and 6845 were silent mutations and did not alter protein translation. Based on the $\mathrm{C}$-to- $\mathrm{U}$ transition rate over 20 clones, the editing level at the normal editing site 6666 was $60 \%$ by APOBEC- 1 alone and hypermutation was $35 \%$ at site 6802 and $5 \%$ at sites 6639 and 6648 . Coexpression of ACF and APOBEC-1 increased editing to $90 \%$ at site 6666 and hypermutation to $70 \%$ at $6802,30 \%$ 


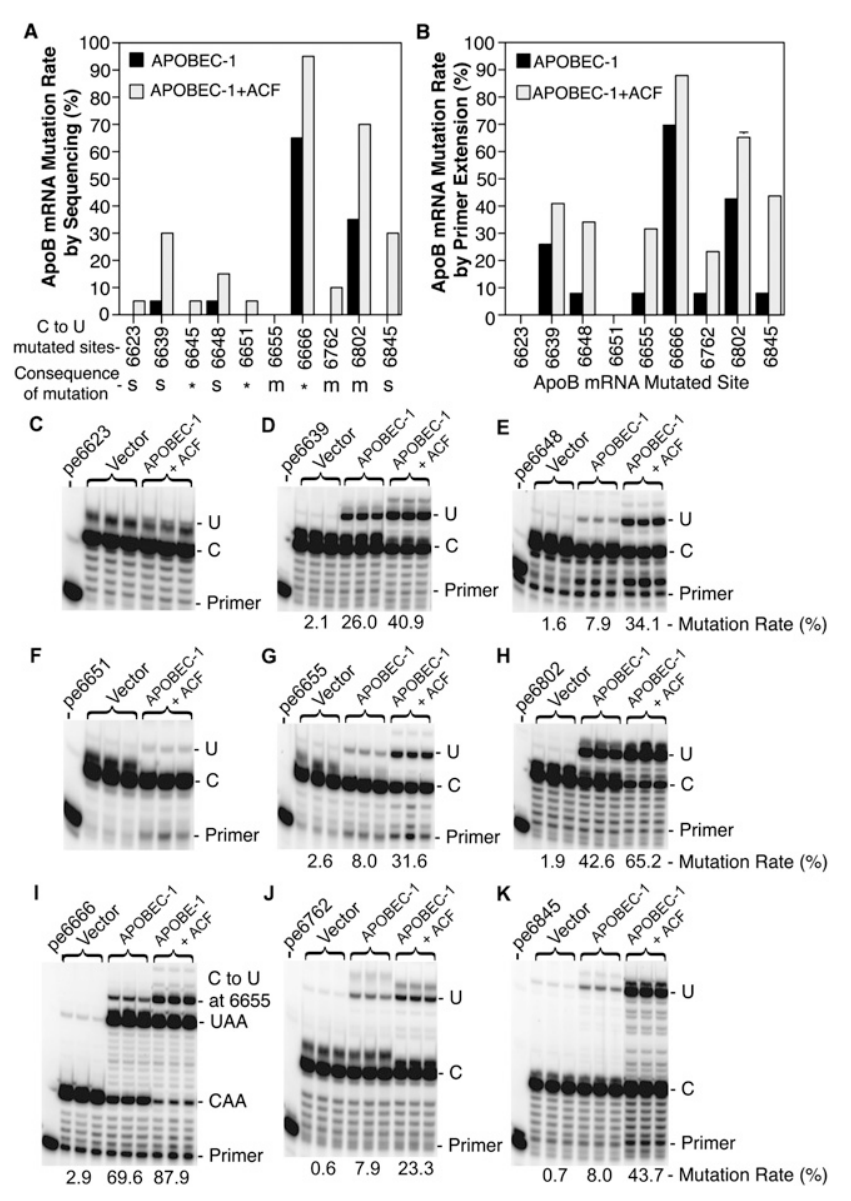

FIGURE 1. Hypermutation site detection by sequencing analyses and verification by primer extension. Adenoviral APOBEC-1 was expressed in HepG2 cells with or without coexpression of adenoviral ACF and total RNAs were extracted after a 2-d transduction. (A) Graphic summary of C-to- $\mathrm{U}$ mutation sites and rates determined by sequencing analyses: The apoB mRNA fragment covering the editing site (6471$6886 \mathrm{nt}$ ) was amplified by RT-PCR and cloned into a pENTR1A vector and the mutation sites were detected by sequencing 20 clones. The mutation rates were determined by occurrence rate at the sites according to the sequencing data. For the consequence of C-to-U transition at each site, ${ }^{*}$, protein translation stop; s, silent mutation; and $\mathrm{m}$, mutation occurred on protein translation. (B) Graphic summary of mutation rates at different sites determined by primer extension: The apoB mRNA fragment was amplified by RT-PCR and the apoB amplicons were subjected to primer extension analyses with individual sense or antisense primer specific for a given site. The resultant primer extension products were separated by $8 \%$ polyacrylamide urea gel electrophoresis and quantified using a PhosphorImager. Each bar represents the mean and standard deviation with $n=3$. $(C-K)$ Primer extension analyses of sites $6623,6639,6648,6651,6655,6666,6762,6802$, and 6845 , respectively. The pe6639 designation stands for the primer extension assay at site 6639 , etc. The hypermutations or editing levels under each assay gel picture were an average of the triplicate samples.

at 6639 , and $15 \%$ at 6648 . This coexpression also caused detectable hypermutation at additional sites including $30 \%$ at $6845,10 \%$ at 6762 , and $5 \%$ at 6623,6645 , and 6651 . In addition, when comparing the distribution of C-to- $\mathrm{U}$ transitions among clones, we also observed that editing at the normal site 6666 was nearly always observed with or without hypermutation occurring at other sites. Only one out of 20 clones had a C-to-U change at site 6802 without a C-to-U transition at site 6666 . These data suggest that site 6666 has greater accessibility/affinity to the editing complex compared to other sites. This is consistent with the mooring sequence being near site 6666 and the mooringlike sequence being near site 6802 and the proposal that canonical editing by the editing complex is associated with mooring motif recognition (Hersberger and Innerarity 1998; Maris et al. 2005).

The sequencing analysis of PCR amplified apoB RNA fragment above is based on a limited number of clones and potentially includes errors due to DNA polymerase PCR reaction. To further verify hypermutation at these sites and obtain a quantitative evaluation of hypermutation, primer extension analyses were performed. Primers were designed for each individual site as described in the Materials and Methods section, except for site 6645 which had cytidines too close to each other to be clearly separated. As shown in Figure 1, C-K, hypermutation at sites 6639, 6648, 6655, 6762,6802 , and 6845 was confirmed by their correlation of the C-to-U mutation levels with the treatment by APOBEC-1 or APOBEC-1 plus ACF. However, primer extension at sites 6623 and 6651 showed background levels only or had no significant differences corresponding to the treatments (Fig. 1C,F), indicating that they were false positive sites. As shown in Figure 1, I, H, and D, APOBEC-1 alone resulted in $69.6 \%$ editing at site 6666 and $42.6 \%$ and $26.0 \%$ hypermutation at sites 6802 and 6639 , respectively. APOBEC-1 alone also induced about $8 \%$ hypermutation at sites 6648, 6655, 6762, and 6845 (Fig. 1E,G,J,K). Coexpression of ACF and APOBEC-1 increased editing and hypermutation significantly at all sites. ACF increased APOBEC-1 editing at site 6666 from $69.6 \%$ to $87.9 \%$ and hypermutation at 6802 from $42.6 \%$ to $65.2 \%, 6639$ from $26.0 \%$ to $40.9 \%$, 6648 from $7.9 \%$ to $34.1 \%, 6655$ from $8.0 \%$ to $31.6 \%, 6762$ from $7.9 \%$ to $23.3 \%$, and 6845 from $8.0 \%$ to $43.7 \%$ as summarized in Figure 1B. These data demonstrate that ACF increases editing and hypermutation induced by APOBEC-1 overexpression with variable site sensitivity.

\section{APOBEC-1 is a determinant factor for hypermutation}

APOBEC-1 hypermutation activity at various sites was differentially affected by ACF as described above (Fig. $1 A, B)$. To further investigate their contribution to hypermutation, the effect of APOBEC-1 and ACF at different expression levels was evaluated. As shown in Figure 2, A-C, hypermutation and editing increased dose-dependently with the amount of viral loading of APOBEC-1 and ACF. The hypermutation response also varied significantly at different sites. Hypermutation increased about 1.5-2.2-fold with sites $6639,6648,6655$, and 6845 when APOBEC-1 was increased from 5 to $100 \mu \mathrm{L}$ regardless of whether 5,25 , or $100 \mu \mathrm{L}$ of ACF were coexpressed. Meanwhile, hypermutation at site 


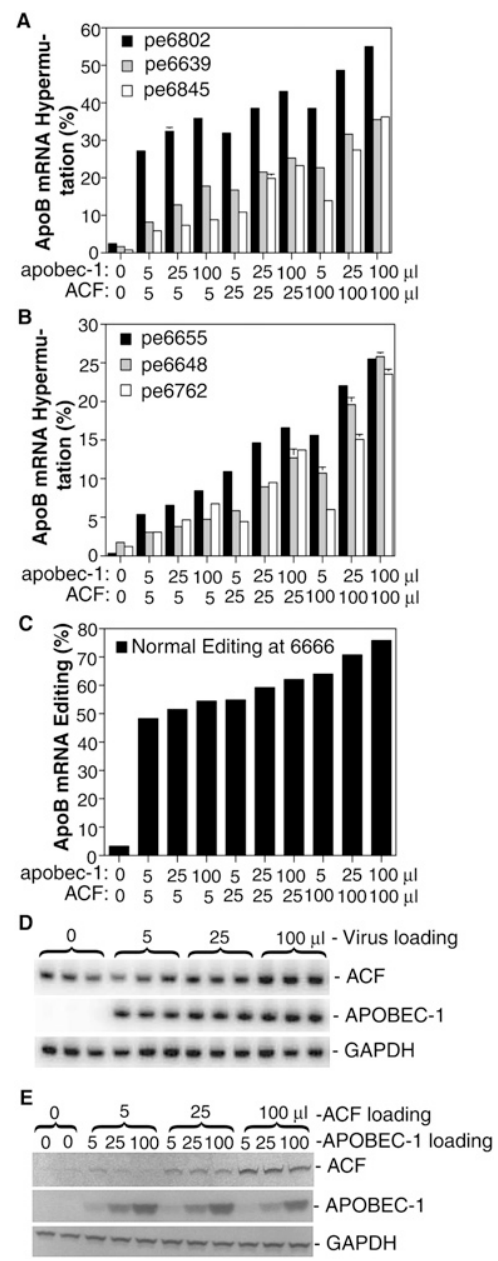

FIGURE 2. ACF dose dependently increases APOBEC-1 hypermutation. Increasing amounts of ACF and APOBEC-1 adenovirus were added to HepG2 cells and total RNAs were extracted after a 2-d transduction. ApoB mRNA was amplified by RT-PCR and the purified amplicons were subjected to extensive primer extension analyses for each individual site followed by gel separation and quantitation with a PhosphorImager. $(A-C)$ Data are summarized and graphically presented with hypermutation or editing sites indicated. The control was HepG2 cells treated with expression gene null adenovirus. The pe6639, etc., designation represents the primer extension assay at site 6639, etc. Each bar represents the mean and standard deviation with $n=3$. (D) mRNA expression analyses of ACF and APOBEC-1 relative to GAPDH: cDNA aliquots underwent semiquantitative PCR amplification in the presence of $\left[\alpha-{ }^{32} \mathrm{P}\right]$-dCTP and the abundance of each gene was determined according to their PCR product counts after separation with $6 \%$ polyacrylamide gel electrophoresis. $(E)$ Protein expression analyses of ACF and APOBEC- 1 by Western blotting: HA tagged APOBEC- 1 and FLAG tagged ACF in adenovirus were expressed in HepG2 cells. Expressed proteins were harvested by lysing cells in SDS sample buffer and separated on 12\% SDS-PAGE gel electrophoresis followed by Western detection with antibodies against HA tag or native ACF. GAPDH was taken as reference for the comparison of protein sample loading.

6762 increased 2.2-, 3.1-, and 3.9-fold, depending on an ACF loading of 5, 25, or $100 \mu \mathrm{L}$, respectively. These data indicate that APOBEC-1 may play a determinant role for hypermutation. The mRNA and protein expression levels of APOBEC-1 and ACF all increased dose-dependently when the viral loading increased from 5 to 25 or $100 \mu \mathrm{L}$ (Fig. 2D,E). Compared to endogenous ACF levels, ACF protein expression was increased $8-10$-fold by the $100 \mu \mathrm{L}$ viral loading. The expressed ACF, as evaluated by confocal microscopy, was localized in both the nucleus and cytoplasm, primarily in the cytoplasm (data not shown). The viral titers for these adenoviral preparations were about $5 \times 10^{8} \mathrm{pfu} / \mathrm{mL}$.

Alternatively, when ACF was increased from 5 to $100 \mu \mathrm{L}$, hypermutation increased 2.0-5.4-fold depending on different APOBEC-1 loadings of 5, 25, or $100 \mu \mathrm{L}$ (Fig. 2A,B). Increasing $\mathrm{ACF}$ from 5 to $100 \mu \mathrm{L}$ resulted in 3.5-, 5.1-, or 5.4 -fold increases at site 6648 , corresponding to 5,25 , or $100 \mu \mathrm{L}$ of APOBEC-1 coexpression, respectively. Meanwhile, a 2.8-, 2.5-, or 2.0-fold increase was observed at site 6639 under the same conditions. These data indicate that ACF has a stimulating effect on APOBEC-1 hypermutation with a variable response by different sites and that higher ACF concentrations usually result in higher hypermutation.

The normal editing at site 6666 and the major hypermutation site 6802 were also dose-dependently increased about 1.3- and 1.5-fold, respectively. These increases were not as large as observed in the other sites, since they were limited by pre-existing high editing levels (Fig. 2A,C). These data indicate that the editing at normal site 6666 and hypermutation at site 6802 have a higher accessibility/ affinity to the APOBEC-1 and ACF complex because of the presence of downstream mooring or mooring-like motifs and are probably saturated within the testing concentration range of APOBEC-1 and ACF. Larger amounts of APOBEC-1 or ACF do not further increase normal editing, but generate additional hypermutation.

APOBEC-1 is normally expressed at very low levels under physiological conditions, while ACF is expressed more abundantly. We wondered if the aberrant APOBEC-1 hypermutation activity could be minimized under conditions of low level APOBEC-1 and abundant ACF expression. As shown in Figure 3, APOBEC-1 hypermutation activity became undetectable at the major hypermutation site 6802 when APOBEC-1 viral loading was decreased to below $0.9 \mu \mathrm{L}$ (Fig. 3B), while normal editing was still detectable even down to $0.1 \mu \mathrm{L}$ (Fig. 3A). When ACF $(100 \mu \mathrm{L})$ was coexpressed together with APOBEC-1 under the same viral loading from 0.1 to $3.0 \mu \mathrm{L}$, normal editing increased 2.23.8-fold with the greater magnitude of increase observed with lower APOBEC-1 viral loading (Fig. 3A,C). APOBEC-1 hypermutation activity was also increased and became detectable at $0.3 \mu \mathrm{L}$ instead of $0.9 \mu \mathrm{L}$ in the presence of ACF (Fig. 3B,D). A fourfold hypermutation increase by ACF was observed with $3.0 \mu \mathrm{L}$ APOBEC-1. Hypermutation at site 6655 detected by appearance of an additional band above UAA in Figure $3 \mathrm{~A}$ was parallel to the hypermutation at site 6802 in Figure $3 \mathrm{~B}$ and provided further evidence for the observed hypermutation activity. These data confirm that hypermutation is dependent on the expression levels of 


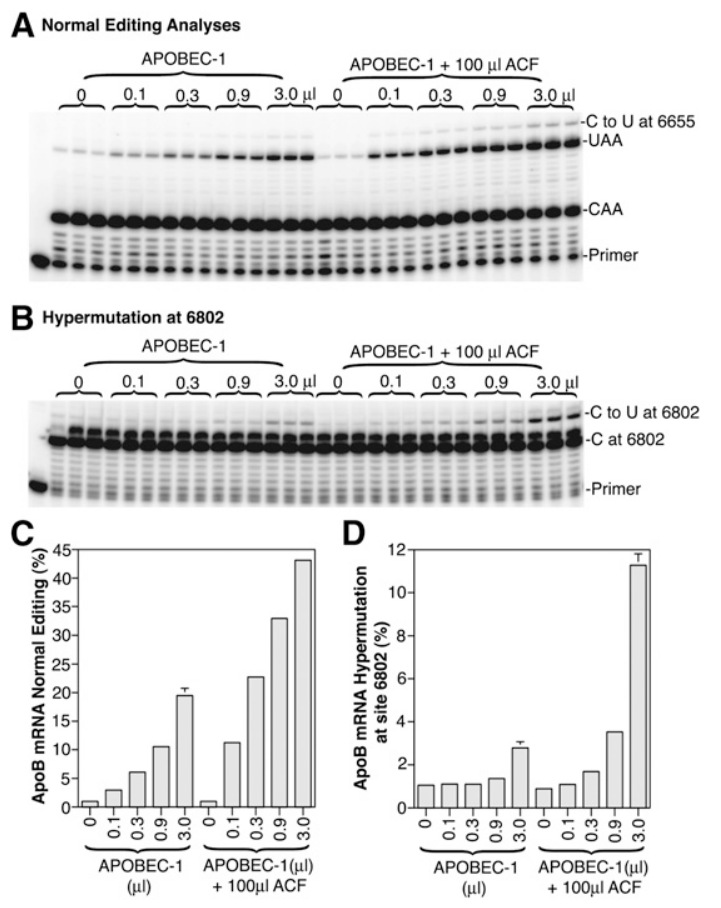

FIGURE 3. ACF promotes occurrence of hypermutation with low levels of APOBEC-1. Increasing amounts of APOBEC-1 adenovirus were added to HepG2 cells with or without ACF adenovirus and total RNAs were extracted after a 2-d viral exposure. ApoB mRNA was amplified by RT-PCR. With the purified amplicons, $(A)$ normal editing at site 6666 and $(B)$ a representative hypermutation at site 6802 were determined by primer extension. $(C, D)$ Data were further summarized and graphically presented with normal editing or hypermutation indicated. Each bar represents the mean and standard deviation with $n=3$.

APOBEC-1 and further suggest that abundant levels of ACF have a stimulating effect on APOBEC-1 hypermutation.

\section{The single point APOBEC-1 mutants, P29F and $E 181 Q$, eliminate hypermutation}

In vitro structure and function studies have shown that rat APOBEC-1 has four regions essential for apoB mRNA editing, including basic amino acid clusters in the aminoterminal region (R15-K34), a catalytic domain (H61, E63, C93, C96), a leucine-rich motif (L180-L196), and a dimerization domain in the carboxyl-terminal region (Teng et al. 1999). The basic amino acid cluster (R15-17, R33K34) and P29 have been proposed to be crucial for APOBEC-1 nuclear localization through interaction with importin $\alpha$ (Chester et al. 2003). The P29T mutation abolished APOBEC-1 in vitro editing activity and the protein interaction with importin $\alpha$ (Chester et al. 2003). To further investigate the role of APOBEC-1 in hypermutation, we first tested the $\mathrm{N}$-terminal basic amino acid cluster mutants, R17A, P29F, R33A, K34A, and R33K34A and catalytic domain mutants, H61A and E63Q, by adenoviral overexpression in HepG2 cells in the presence of ACF. Their effects were evaluated by their induced editing and hypermutation activities on apoB mRNA with primer extension at two major representative sites, 6639 and 6802 as identified in Figure 1.

As shown in Figures 4 and 5, APOBEC-1 mutation significantly affected hypermutation activity. The catalytic site mutant, H61A decreased normal editing activity from $87.9 \%$ to $31.5 \%$, while its adjacent mutant V62A still had $80.8 \%$ editing (Figs. 4A, 5A). The H61A mutation decreased all hypermutation to background levels, but V62A had hypermutation levels close to wild-type levels (Figs. 4B,C, 5). The E63Q mutation completely lost both editing activity at the normal site 6666 and hypermutation at all sites, consistent with the proposal that E63 plays a critical role in the deamination process of cytidine (Yamanaka et al. 1994). The inactive E63Q mutant could be taken as an internal reference of background for other APOBEC-1 mutant analyses. Compared to wild-type APOBEC-1 control, the P29F mutation decreased hypermutation at sites 6802, 6639, and 6655 to background levels, while retaining $47.2 \%$ editing at the normal site 6666 as shown in Figure 4, A-C, and Figure 5. The K34A mutant had an effect similar to P29F except for $8.1 \%$ and $2.3 \%$ hypermutation remaining at sites 6802 and 6639 , respectively. The R33K34A double mutant eliminated all hypermutation but also decreased the editing from $87.9 \%$ to $20.4 \%$. The R17A and R33A mutations also decreased editing and hypermutation, but significant hypermutation activity remained at sites 6802 and 6639. These data indicate that the P29F mutation selectively abolished APOBEC-1 hypermutation activity, while maintaining $47.2 \%$ editing activity at the normal editing site in the presence of ACF overexpression. In the absence of ACF overexpression, the P29F mutant still had 39.6\% editing with no observable hypermutation (data not shown).

The basic character of the APOBEC-1 basic amino acid cluster region made us wonder if it could potentially interact with acidic amino acids in other APOBEC-1 regions and what role the consensus leucine-rich motif (L180-P191) has in APOBEC-1 hypermutation. We evaluated the E181Q mutant that eliminated acidic amino acids and mutants of leucine-rich region, including L180F, L182A, I185A, L187A, L189A, and P190P191A. As shown in Figures 4 and 5, the E181Q mutation decreased hypermutation to background levels at all sites while retaining $46.7 \%$ editing at the normal site 6666. The hypermutation activity with E181Q was not detected in HepG2 cells even with prolonged viral expression up to $4 \mathrm{~d}$ with or without ACF coexpression (data not shown). These data indicate that like P29F, E181Q selectively decreased APOBEC-1 hypermutation to background levels, while retaining half of the wild-type normal editing. The L182A and P190A/P191A mutants had an effect similar to E181Q with decreased hypermutation to near background levels at sites 6639 and 6655, but still had 9.4\% and 10.1\% hypermutation activity at site 6802 , while retaining $50.5 \%$ and $44.9 \%$ normal editing activity, respectively. The L187A mutant was similar to wild-type APOBEC-1. The I185A and 


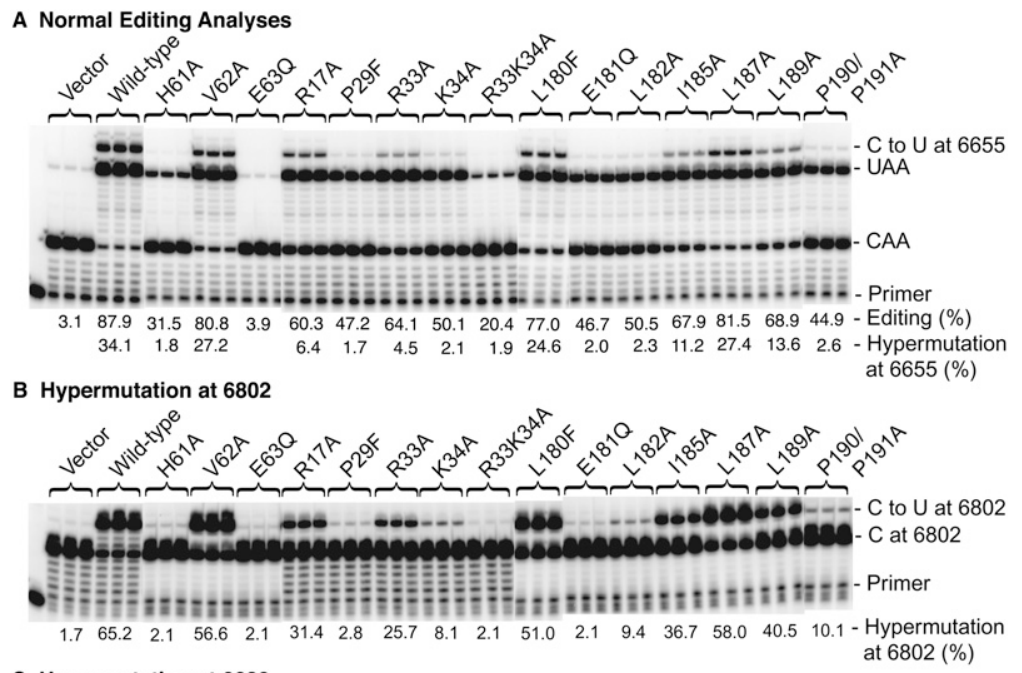

C Hypermutation at 6639

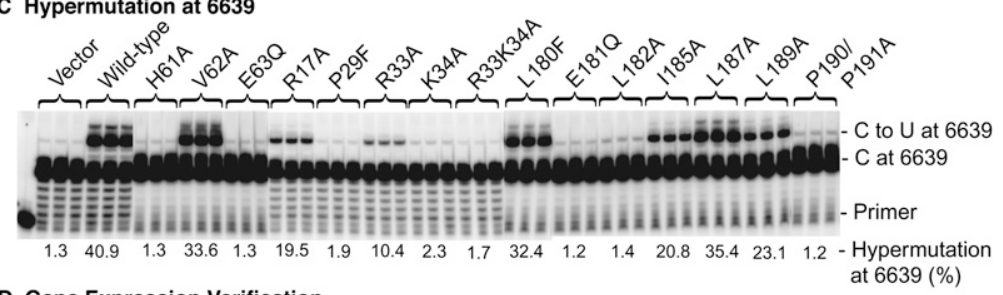

D Gene Expression Verification

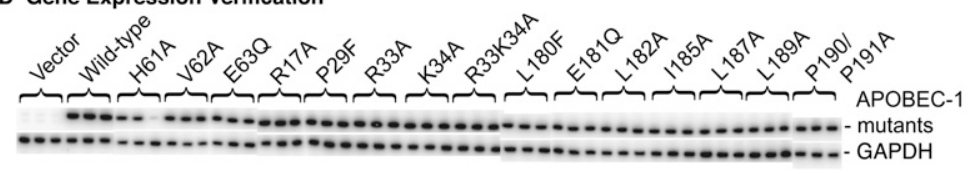

FIGURE 4. Effect of APOBEC-1 mutations on apoB hypermutation in HepG2 cells. APOBEC-1 mutants encoded in adenovirus were expressed in HepG2 cells in the presence of ACF adenovirus and total RNAs were extracted after a 2-d viral exposure. ApoB mRNA was amplified by RT-PCR. (A) Normal editing at site 6666 was determined by primer extension assay and $(B, C)$ representative hypermutation at sites 6802 and 6639 was determined, respectively. The editing or hypermutation levels were an average of triplicate samples determined by a PhosphorImager after primer extension and gel separation. The gel presentation was a combination from three separated gels under the same setting. $(D)$ mRNA expression verification of mutant genes versus house keeping gene GAPDH: cDNA aliquots underwent semiquantitative PCR amplification in the presence of $\left[\alpha-{ }^{32} \mathrm{P}\right]$-dCTP and the abundance of each gene was determined according to their PCR product counts after separation with $6 \%$ polyacrylamide gel electrophoresis.

L189A mutations decreased editing and hypermutation, but significant hypermutation activity remained. These data demonstrate that hypermutation associated with APOBEC-1 overexpression can be eliminated by a single point mutation of $\mathrm{P} 29 \mathrm{~F}$ or E181Q.

We evaluated mRNA expression levels of all APOBEC-1 mutants expressed in the HepG2 cells (Fig. 4D) and protein levels of selected mutants including P29F, K34A, E63Q, E181Q, L182A, and P190P191A (data not shown). As shown in Figure 4D, mRNA levels were comparable to wild type for all mutants after normalization with the house keeping gene GAPDH. For protein levels, all mutants were also comparable except for E63Q and P190P191A, which decreased about $70 \%$ and $50 \%$, respectively, compared to wild-type control. However, in comparing normal editing and hypermutation activities over a wide range of expressed APOBEC-1 as shown in Figure 2, a similar pattern was observed although there were some differences in magnitude. These results support an interpretable semiquantitative comparison despite expression variation with some of the mutants.

\section{The APOBEC-1 mutants, P29F and $E 181 Q$, edit apoB mRNA without hypermutation in primary cultured rat liver cells}

The P29F and E181Q mutants eliminate hypermutation on apoB mRNA, while retaining about half of the normal wildtype editing in HepG2 cells. We wondered how they functioned in rat primary cultured hepatocytes, a situation closer to actual animal testing. NAT1 (novel APOBEC-1 target 1) has been identified as a hypermutation target gene in mouse liver overexpressing APOBEC1 (Yamanaka et al. 1997). We evaluated if mutant overexpression would edit apoB mRNA without NAT1 hypermutation. Primary hepatocytes from rat liver were isolated and APOBEC-1 mutants were expressed overnight in the presence of ACF. Rat, instead of mouse, liver was used because mouse liver was too small to obtain sufficient numbers of hepatocytes. As shown in Figure 6A, rat endogenous apoB mRNA had 75\% natural editing at site 6479 (equivalent to site 6666 of human apoB). Adenoviral expression of wild-type APOBEC-1 and most mutants together with ACF coexpression resulted in greater than 97\% editing. The editing inactive mutant E63Q and partially inactive mutant H61A decreased apoB mRNA editing from $75 \%$ to $51 \%$ and $57 \%$, respectively, indicating that E63Q and H61A mutants were competitive with endogenous APOBEC-1 and resulted in domain inhibition. The R33K34A mutant had an inhibiting effect similar to E63Q, decreasing editing from $75 \%$ to $59 \%$. The P29F, E181Q, and P190P191A mutants had 83\%, 79\%, and $82 \%$ editing, respectively, indicating they had an intermediate editing activity compared to the wild-type control of $97 \%$. It should be noted that the adenovirus null vector control also resulted in $95 \%$ editing. The reason for this effect remains to be clarified. We evaluated the mRNA expression levels of editing components by quantitative PCR and found that rat APOBEC-1 levels were comparable. However, ACF levels were decreased about $50 \%$ by empty 


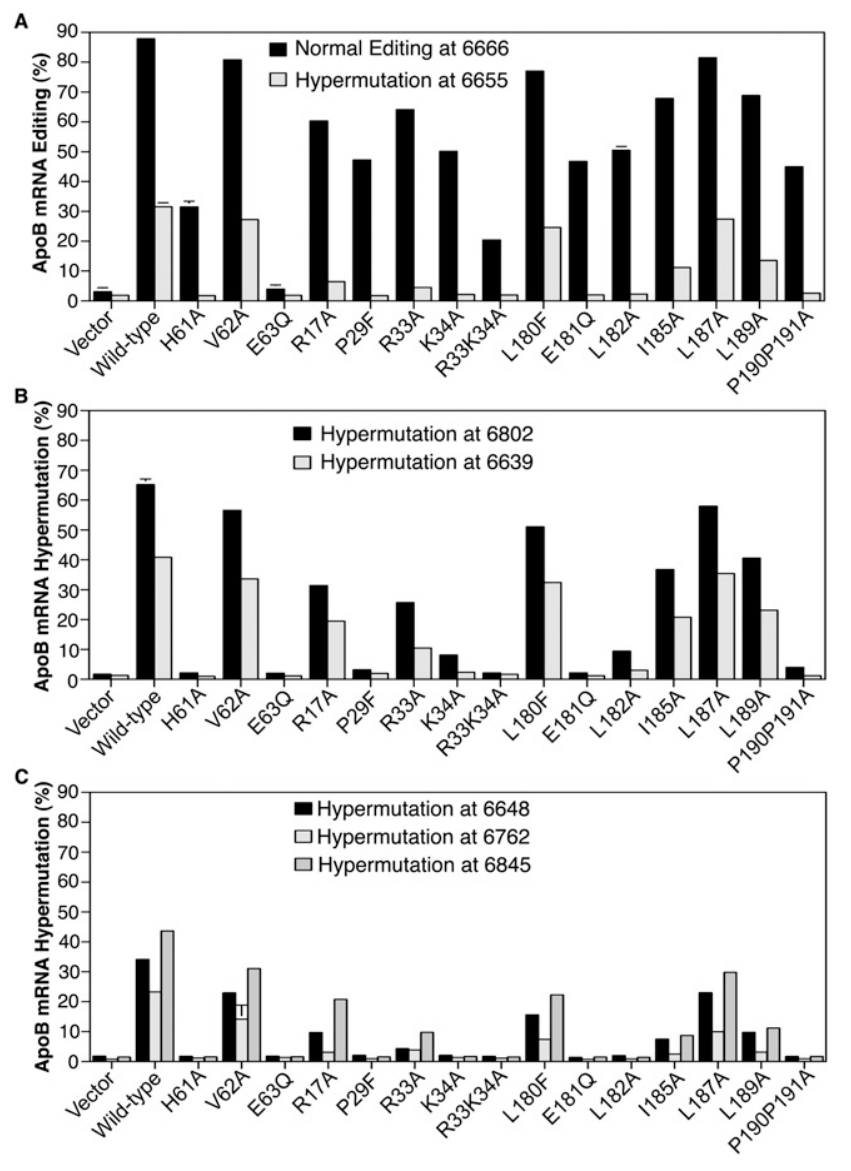

FIGURE 5. Effect of APOBEC-1 mutations on apoB hypermutation: Graphic representation. As in Figure 4, the apoB RT-PCR amplicons were purified and subjected to extensive primer extension analyses for each individual site including 6639, 6648, 6655, 6666, 6762, 6802, and 6845 . The data were summarized and presented in graphs. (A) Normal editing at site 6666 and hypermutation at sites 6655, $(B)$ hypermutation at sites 6802 and 6639 , and $(C)$ hypermutation at sites 6648,6762 , and 6845 . Each bar represents the mean and standard deviation with $n=3$.

adenovirus vector. For determination of normal editing and hypermutation here, both ACF and APOBEC-1s were overexpressed. Consequently there was ample ACF present in these experiments and hypermutation activities were significantly above background (Fig. 6B,C), allowing ready interpretation of APOBEC-1 mutant effects on apoB and NAT1 mRNA.

Hypermutation of rat apoB mRNA induced by APOBEC-1 overexpression in rat liver cell lines, McA7777 and FAO was significantly different than that of human apoB in HepG2 cells. Sequence analyses revealed that cytidine at site 6472 was the highest hypermutation site on rat apoB mRNA (data not shown). Thus, primer extension against site 6472 was analyzed to determine hypermutation of rat apoB mRNA in the rat primary hepatocytes. As shown in Figure $6 \mathrm{~B}$, blank control, adenovirus null vector control, and the editing inactivated mutant E63Q gave background levels of $5.7 \%, 9.4 \%$, and $6.5 \%$, respectively. Wild-type APOBEC-1 expression increased hypermutation up to $18.4 \%$. E181Q decreased hypermutation to $5.3 \%$, a background level. P29F had 7\%, an effect similar to E181Q. These data suggest that APOBEC-1 mutants have similar effects on apoB mRNA editing and hypermutation in both human HepG2 cells and rat primary hepatocytes.

Hypermutation of NAT1 mRNA by primer extension at site 3736 has been developed as a means to evaluate the hypermutation effect of APOBEC-1 overexpression on a gene other than apoB in mouse liver (Yamanaka et al. 1997). The NAT1 gene is highly conserved across different species and identical RT-PCR amplifications were obtained for the NAT1 gene region around site 3736 with rat hepatocytes. As shown in Figure 6C, blank control, adenovirus null
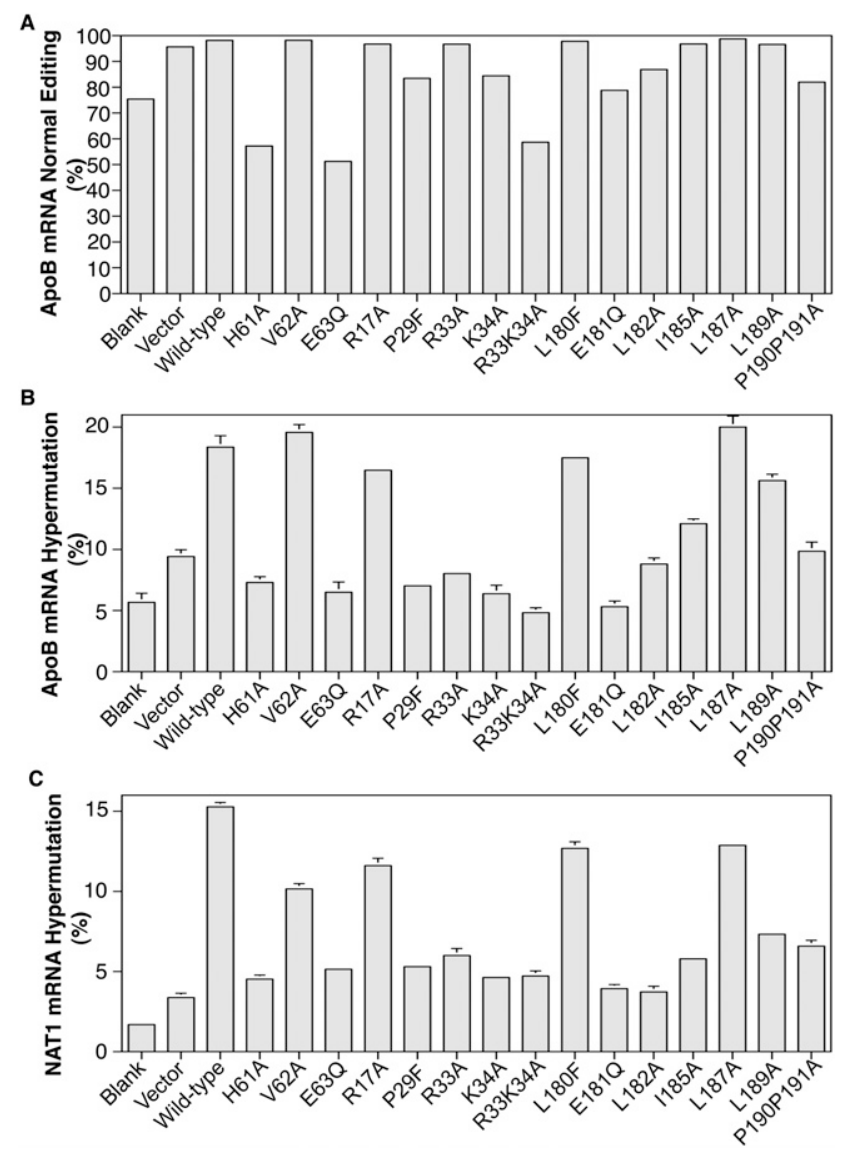

FIGURE 6. Effect of APOBEC-1 mutation on hypermutation of NAT1 (novel Apobec-1 target no. 1) in rat liver primary cultured hepatocytes. Hepatocytes were isolated from rat liver. APOBEC-1 mutants encoded in adenovirus were expressed in the primary cultured cells together with adenoviral ACF and total RNAs were extracted after overnight viral exposure. The apoB and NAT1 fragments covering the editing or hypermutation sites were PCR amplified from cDNA. The editing or hypermutaion effects of the APOBEC-1 mutants were determined by primer extension of the PCR amplicons followed by separation with $8 \%$ polyacrylamide gel electrophoresis. The data from each individual primer extension were summarized and presented as $(A)$ rat apoB mRNA normal editing; $(B)$ rat apoB mRNA hypermutation at site 6472; and $(C)$ rat NAT1 mRNA hypermutation at site 3736 . Each bar represents the mean and standard deviation with $n=3$. 
vector control, and the editing inactivated mutant E63Q had background levels of $1.7 \%, 3.4 \%$, and $5.1 \%$, respectively. Expression of wild-type APOBEC-1 increased hypermutation up to $15.3 \%$. E181Q and P29F decreased hypermutation to background levels, $3.9 \%$ and $5.3 \%$, respectively. The overall pattern of hypermutation changes in NAT1 gene in response to different APOBEC-1 mutation was comparable to that of apoB in rat hepatocytes and human HepG2 cells (Figs. 5, 6B). These data suggest that the APOBEC-1 mutants, E181Q and P29F, not only eliminate hypermutation on apoB mRNA, but also other potential targets like NAT1, while retaining reduced normal apoB mRNA editing activity.

\section{The decreased hypermutation of APOBEC-1 mutants is associated with decreased protein interaction with ACF}

It has been reported that the basic amino acid cluster R15-17 and R33K34 of APOBEC-1 is a site for protein interactions between APOBEC-1 and ACF. The $\mathrm{P} 29 \mathrm{~T}$ mutation in this region abolishes this interaction (Chester et al. 2003). We found that the APOBEC-1 mutants, P29F and E181Q, resulted in no hypermutation, while retaining half of normal specific editing, suggesting that eliminating hypermutation may be due to a lost or decreased protein interaction between the APOBEC-1 mutant and ACF. To evaluate this mechanism, we investigated the correlation between protein interaction and APOBEC-1 hypermutation activity. APOBEC-1 mutants with or without a HA tag were cotranslated with FLAG tagged ACF in an in vitro coupled transcription and translation system followed by immunoprecipitation with an antibody against ACF-FLAG. The level of protein interaction between APOBEC-1 mutants and ACF was determined by the amount of APOBEC-1 mutant proteins coprecipitated with ACF (Teng et al. 1999).

As shown in Figure 7, A-C, all APOBEC-1 mutants had comparable protein levels in the cotranslation mixture with ACF. After immunoprecipitation with ACF, the protein levels varied with different APOBEC-1 mutants, reflecting their different protein interactions with ACF. Mutants of P29F, E63Q, E181Q, L182A, L182E, and P190P191A de-
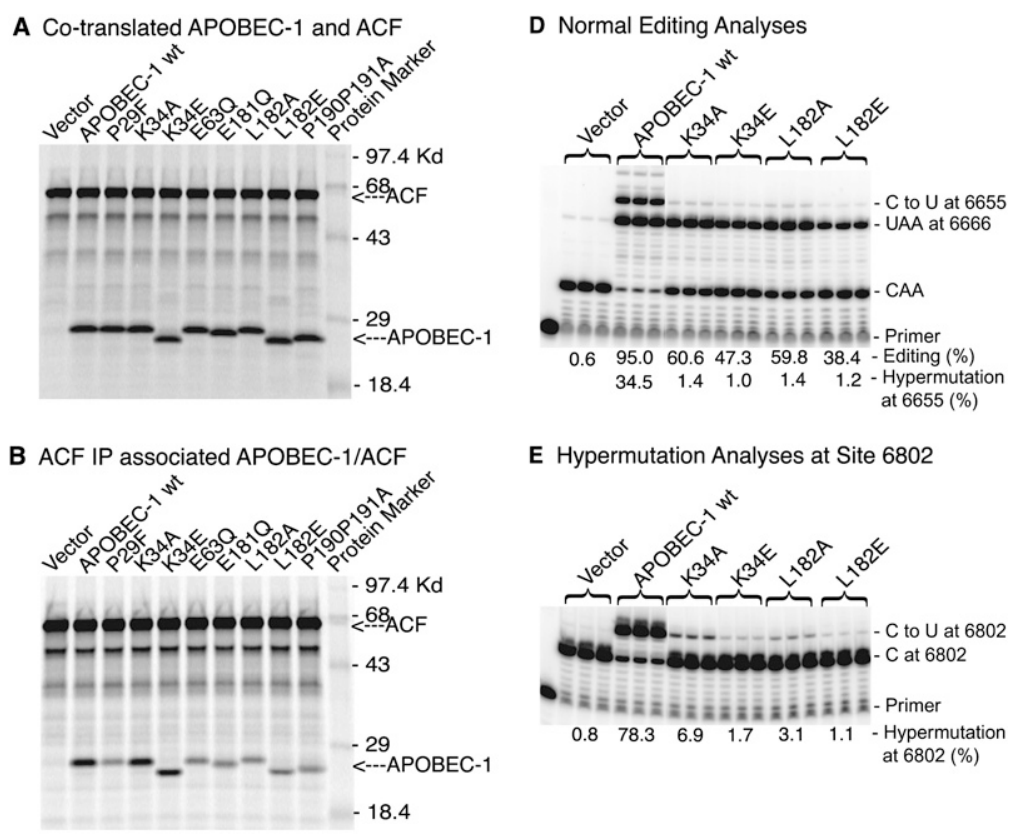

E Hypermutation Analyses at Site 6802

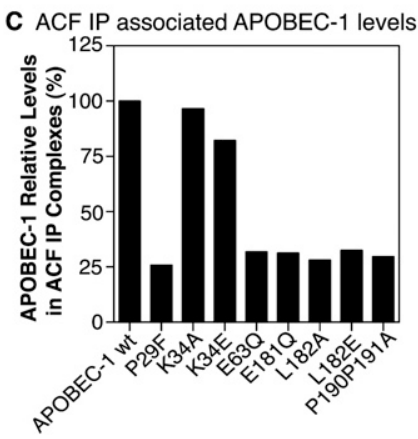

F Hypermutation Analyses at Site 6639

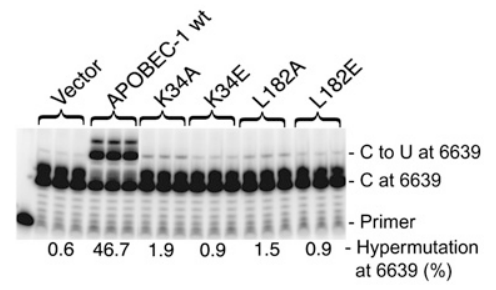

FIGURE 7. The decreased hypermutation activity of APOBEC-1 mutants is associated with decreased protein interaction with ACF. APOBEC-1 mutants with or without a HA tag were cotranslated with FLAG tagged ACF in a coupled transcription/translation system in the presence of $\mathrm{L}-^{35} \mathrm{~S}$-methionine. The protein complexes formed during the in vitro translation were immunoprecipitated by an antibody against the FLAG tag. The APOBEC-1 proteins associated with ACF were separated on a 12\% SDS-PAGE denaturing gel and were determined by a PhosphorImager. As a control for each protein translation, an aliquot before immunoprecipitation was denatured directly in SDS-sample buffer and separated on a 12\% SDS-PAGE gel in parallel. $(A)$ APOBEC-1 mutant proteins cotranslated with ACF; $(B)$ APOBEC-1 mutant proteins immunoprecipitated with ACF; and $(C)$ Quantitative presentation of immunoprecipitated APOBEC-1 mutant proteins in $B .(D-F)$ Editing and hypermutation activity as determined in Figure 4 for additional APOBEC-1 mutants, K34E and L182E to compare with K34A and L182A. Normal editing at site 6666 was determined by primer extension analyses in $D$ and representative hypermutation activity at sites 6802 and 6639 was determined in $E$ and $F$, respectively. The hypermutation or editing levels were an average of triplicate samples.

creased to $\sim 30 \%$ of the wild-type control. The decreased protein interaction of these mutants paralleled their decreased hypermutation activities, which were at or near background levels (Figs. 4, 5), suggesting that a decreased protein interaction with ACF may play an important role in their diminished hypermutation activity. In contrast, the K34A mutant also had significantly decreased hypermutation activity (for example, as shown in Fig. 7E, from $78.3 \%$ to $6.9 \%$ at site 6802 ), but the coprecipitated $\mathrm{K} 34 \mathrm{~A}$ protein was almost identical with the wild type, suggesting a different 
mechanism for diminished hypermutation than just the APOBEC-1/ACF interaction. To better understand this, we further tested different mutations for K34 and L182. As shown in Figure 7, D-F, the K34A and L182A mutants had $6.9 \%$ and $3.1 \%$ hypermutation activity at site 6802 . By further disrupting the protein interaction with a charge change or increased residue size, the K34E and L182E mutants decreased their hypermutation activities to near background levels. In addition, the K34E mutation also had a decreased protein interaction to $82 \%$ of wild type. The additionally reduced hypermutation activity from K34A to K34E or from L182A to L182E, with additional protein disruption, further supports the proposal that a decreased protein interaction between APOBEC-1 and ACF may play an important role in decreased hypermutation activity.

\section{DISCUSSION}

APOBEC-1 overexpression in liver can reduce atherogenic LDL levels and potentially could be used for the treatment of hyperlipidemia. However, overexpression of APOBEC-1 causes hypermutation that is believed to induce liver cancer through nonspecific mRNA editing, thus raising a significant safety concern of APOBEC-1 as a therapeutic agent. Although apoB mRNA editing has been studied extensively, how to overcome hypermutation remains unknown. ApoB mRNA editing is exquisitely precise under physiological conditions and editing is performed by APOBEC- 1 and cofactors potentially including ACF, CUGBP2, GRY-RBP, KSRP, hnRNP-C1, ABBP1, ABBP2, and BAG4. How these proteins interact to achieve the editing specificity remains largely unknown.

We reasoned that overexpression of APOBEC-1 in human liver HepG2 cells provides an in vitro cellular model for the in vivo human situation of APOBEC-1 overexpression in the liver. So we used adenoviral expression of APOBEC-1 and its auxiliary cofactors in HepG2 cells to investigate the effect of cofactors and APOBEC-1 structure itself on apoB mRNA hypermutation to determine if the APOBEC-1-induced hypermutation could be eliminated. The near-100\% adenovirus transduction efficiency made it possible to accurately detect hypermutation using a total RNA extract. The adenoviral system also allowed easy manipulation for coexpression of different genes at the same time. We found that overexpression of the core auxiliary factor ACF significantly increased hypermutation and that the APOBEC-1 structure itself was very critical for hypermutation activity. Single point mutations of APOBEC-1, P29F, and E181Q, virtually eliminate hypermutation on both apoB and NAT1, while maintaining half-normal editing of wild-type APOBEC-1, reopening a possible gene therapy approach using APOBEC-1.

ACF plays a critical role in apoB mRNA editing. APOBEC1 alone has no editing activity. ACF and APOBEC-1 form a complex minimally required for in vitro apoB mRNA editing. It has been proposed that ACF first recognizes the flexible nucleotides of the apoB mRNA mooring sequence and subsequently melts the stem-loop, exposing the amino group of cytidine 6666 to APOBEC-1 (Maris et al. 2005). $\mathrm{ACF}$ also lowers the temperature requirement to promote a conformational transition in the RNA substrate (Chester et al. 2004). ACF expression increases simultaneously with APOBEC-1 in small intestine during developmental apoB mRNA editing up-regulation, while inhibitors CUGBP2, GRY-RBP, and hnRNP-C1 decrease (Chen et al. 2007). These data suggest that ACF may play an important role in APOBEC-1 editing efficiency and specificity. However, our data demonstrate that ACF is the major cofactor among the potential cofactors tested that dose-dependently increased hypermutation. The increased effect of ACF cannot be reversed by other editing inhibitory cofactors (data not shown). These data indicate that high levels of ACF compromise the editing specificity of APOBEC-1.

ACF increased both the editing at normal site 6666 and hypermutation at sites $6639,6648,6655,6762,6802$, and 6845. However, the ACF stimulatory effect varied significantly at different sites. For example, hypermutation at the major sites 6802 and 6639 was increased about 1.5 -fold by ACF and normal editing at site 6666 increased 1.3-fold, while hypermutation at sites 6648, 6655, 6762, and 6845 were increased 3-5-fold (Fig. 1B). On the other hand, editing at the normal site was nearly always observed (95\% occurrence) in individual clone sequencing analyses (Fig. 1A). When normal editing at site 6666 was absent, it was rare to observe hypermutation (5\% occurrence). In this case, hypermutation was only observed at site 6802. These data suggest that with the mooring sequence nearby, the normal editing site 6666 is readily accessible by the editing complex and becomes saturated easily with increased availability of editing complex. Hypermutation is not a random event, but rather one that is associated with the normal editing process due to excess APOBEC-1 editing complex. The variable site sensitivity of hypermutation may reflect different site accessibility to the editing complex. Consistent with this, hypermutation at site 6802 was found to be a major one among the hypermutation sites due to its downstream moor-like sequence. The dramatic effect of hypermutation elimination by the single point mutations, P29F and E181Q, demonstrates that APOBEC-1 is a determinant factor for hypermutation. Taken together, these data suggest that low APOBEC-1 concentration and APOBEC-1 associated complex availability may play a major role on apoB mRNA editing specificity under physiologic conditions.

Inducing apoB mRNA editing by APOBEC-1 overexpression results in hypermutation of many additional nontarget sites. It is surprising that a single amino acid mutation of APOBEC-1 by P29F or E181Q eliminates these hypermutations and maintains editing specificity. Both P29F and E181Q mutants had significantly decreased protein interactions with ACF (Fig. 7), suggesting that APOBEC-1 editing 
activity is regulated by a protein interaction with ACF in the editing complex. It is reported that the basic amino acid cluster R15-17 and R33K34 of APOBEC-1 and the basic amino acid cluster K145-R149 of ACF are important for the protein interaction between APOBEC-1 and ACF (Blanc et al. 2003; Chester et al. 2003). We found that P29, located in the basic acid cluster region, had different effects depending on the specific replacement residue. P29F mutation eliminated hypermutation, but P29T was not as effective as P29F (data not shown). This indicates that an increased amino acid residue size at site 29 is more effective in disrupting the protein interaction required for hypermutation. The E181Q mutation eliminated hypermutation by simply replacing a carboxyl acid $(-\mathrm{COOH})$ with an aldeamine $\left(-\mathrm{CONH}_{2}\right)$. This could reflect a reduced protein interaction between the basic amino acid region (K145-R149) of ACF and the acidic part of the glutamic acid at site 181 of APOBEC-1 by minimizing charges.

In summary, we evaluated how editing cofactors and sitedirected APOBEC-1 mutants affected APOBEC-1 hypermutation to better understand hypermutation and determine if it can be overcome. We found that APOBEC-1 hypermutation is increased by ACF, but is dramatically decreased by site-directed mutations of APOBEC-1. The P29F and E181Q mutants virtually eliminated APOBEC-1 hypermutation on both apoB and NAT1 mRNA, while retaining apoB mRNA editing, reopening a possible approach previously abandoned for the treatment of hyperlipidemia.

\section{MATERIALS AND METHODS}

\section{Cell culture}

The human liver cell line HepG2 was purchased from ATCC, and was maintained in EMEM containing $10 \%$ fetal bovine serum and 1X Pen-Strep. 293A cells were purchased from Invitrogen, and were maintained in DMEM containing 10\% fetal bovine serum and 1X Pen-Strep. For adenoviral transduction of cofactor proteins or APOBEC-1 mutants, HepG2 cells were plated on $35 \mathrm{~mm}$, sixwell dish plates precoated with collagen. The next day, media were replaced with $1 \mathrm{~mL}$ fresh medium and different adenoviral preparations were added. After culture overnight in the presence of adenovirus, the media were replaced with $2 \mathrm{~mL}$ fresh growth media. The cells were incubated overnight and total cellular RNA was extracted using the Trizol reagent (Invitrogen). Three separate samples were prepared for each analysis.

Hepatocytes were isolated from rat liver using the method described by Smedsrod et al. (1985), with minor modifications as described by Bocharov et al. (1995). In brief, the liver was perfused with $500 \mathrm{~mL}$ of $\mathrm{Ca}^{2+} / \mathrm{Mg}^{2+}$-free Hank's balanced salt solution (HBSS) followed by a perfusion with $50 \mathrm{~mL}$ of $0.05 \%$ collagenase type $\mathrm{H}$ (Invitrogen) in $\mathrm{Mg}^{2+}$-free HBSS containing $5 \mu \mathrm{M}$ of $\mathrm{CaCl}_{2}$. Hepatocytes were sedimented by repetitive centrifugations for $3 \mathrm{~min}$ at $50 \mathrm{~g}$ in ice-cold HBSS. Hepatocytes were plated on collagen-coated six-well paltes in Welliam's E media containing $10^{-6} \mathrm{M}$ dexamethasone, $8 \mu \mathrm{g} / \mathrm{mL}$ insulin, $10 \% \mathrm{FBS}$, and $1 \mathrm{X}$ PenStrep antibiotics. Three hours after plating, the unattached cells were removed by replacing with $2 \mathrm{~mL}$ fresh media and the attached cells were further cultivated in the fresh media overnight. Hepatocytes were then infected with adenovirus by replacing with $1.5 \mathrm{~mL}$ fresh media containing adenoviral preparation. The cells were incubated overnight at $37^{\circ} \mathrm{C}$ and total cellular RNA was extracted using the Trizol reagent (Invitrogen). Three separate samples were prepared for each analysis.

\section{Adenoviral constructs and expression preparation}

Full-length cDNA of APOBEC-1, ACF, CUGBP2, GRY-RBP, hnRNP-C1, hnRNP-A1, ABBP1, and ABBP2 from human small intestine; KSRP from Caco-2 cells; and BAG4 from BAG4pCMV6-XL5 (Origene) were RT-PCR amplified by AccuPrime Pfx DNA polymerase (Invitrogen) with primers containing a restriction enzyme cutting site at the end (Table 1). For generation of HA tagged APOBEC-1, the reverse primer of APOBEC-1 was replaced with a primer tagged with a nine-amino acid Haemophilus influenza hemagglutinin epitope (HA-1) preceded by a three-alanine spacer (Teng et al. 1999). For FLAG tagged ACF, the forward primer of ACF was replaced with a primer containing DYKDDDDK insert right after methinine at the N-terminal. The amplicons were cloned into pENTR1A vector (Invitrogen) using restriction enzyme sites. After sequencing confirmation, the genes in pENTR1A were transferred into the adenoviral expression vector $\mathrm{pAd} / \mathrm{CMV} / \mathrm{V} 5-\mathrm{DEST}$ (Invitrogen) by recombination following the manufacturer's instructions. The resultant pAd/CMV plasmids containing the target genes were linearized by $\mathrm{Pac} I$ digestion and were transfected into 293A cells by lipofectamine2000 (Invitrogen). Two days after transfection, the 293A cells were passaged into $100 \mathrm{~mm}$ culture dish and were cultivated until $80 \%$ of cells became detached. The cell suspension was then frozen and thawed three times. After centrifugation at $1750 \mathrm{~g}$ for $15 \mathrm{~min}$, the supernatant was used as the gene expression adenoviral preparation. The titers for these adenoviral preparations generally were about $5 \times 10^{8} \mathrm{pfu} / \mathrm{mL}$ and $100 \mu \mathrm{L}$ were added to HepG2 cells on each 35-mm six-well plate for a single test.

For site-directed APOBEC-1 mutation, QuickChange II XL (Stratagene) was utilized to generate human APOBEC-1 mutants in the pENTR1A vector following the manufacturer's instruction. After sequencing confirmation, the mutant genes were transferred into the adenoviral expression vector, pAd/CMV/V5-DEST and the viral preparation was obtained by the procedure as described above. All plasmid constructs were further verified by in vitro protein translation. The expression of genes or APOBEC-1 mutants in cultured cells was confirmed by the detection of their resultant apoB mRNA editing and mRNA levels by semiquantitative RT-PCR in the presence of $0.4 \mathrm{~mL}\left[\alpha-{ }^{32} \mathrm{P}\right]$-dCTP $(3000$ $\mathrm{Ci} / \mathrm{mmol}, 10 \mathrm{mCi} / \mathrm{mL}$ [Amersham]). The protein expression of APOBEC-1 and ACF in their dose-dependent tests was further confirmed by Western blotting analyses using antibodies (Sigma) against HA for HA-APOBEC-1 or native ACF or FLAG for FLAGACF proteins.

\section{ApoB mRNA hypermutation analyses}

To determine hypermutation induced by adenoviral overexpression of APOBEC-1 or APOBEC-1 plus ACF, an apoB mRNA fragment from 6471 to 6886 was amplified by RT-PCR and cloned into the pENTR1A vector using restriction enzyme sites within 
TABLE 1. Oligonucleotide primers used in this study

\begin{tabular}{|c|c|c|c|}
\hline $\begin{array}{l}\text { Gene } \\
\text { name }\end{array}$ & Oligonucleotide primer sequence & $\begin{array}{l}\text { Primer } \\
\text { location } \\
\quad(n t)\end{array}$ & $\begin{array}{c}\mathrm{NCBI} \\
\text { accession }\end{array}$ \\
\hline APOBEC-1 & $\begin{array}{l}\text { S-BamHI: CGCGgatcccagaggaggaagtccag } \\
\text { AS-EcoRI: CCGGAATTCcatcctattcatctccaagc } \\
\text { HA-AS-EcoRI: CCGGAATTCCtcaagcgtaatctggaacatcgtatg } \\
\text { ggtaagcagcagctctccaagccacagaagg }\end{array}$ & $\begin{array}{c}1-22 \\
734-753\end{array}$ & NM_001644 \\
\hline ACF & $\begin{array}{l}\text { S-BamHI: CGCGGATCctgataatcactctcaatggaatc } \\
\text { AS-Notl: AAGAATGCGGCCGCtaatagagtttgtgtgtc } \\
\text { FLAG-S-BamHI: CGCGGATCCGTGGCGGCCGCCAC } \\
\text { CATGGATTACAAGGATGACGACGATAAGgaatcaaatcacaaatccg }\end{array}$ & $\begin{array}{c}173-196 \\
1976-1994\end{array}$ & NM-014576 \\
\hline CUGBP2 & $\begin{array}{l}\text { S-Kpnl: CGGGGTACCCatttcctcccggacatgacgg } \\
\text { AS-Notl: AAGAATGCGGCCGCtaggatcagtaaggtttgctgtcg }\end{array}$ & $\begin{array}{c}21-41 \\
682-706\end{array}$ & NM_006561 \\
\hline GRY-RBP & $\begin{array}{l}\text { S-Kpnl: CGGGGTACCCtcagcccaacggggagatctctgg } \\
\text { AS-Notl: AAGAATGCGGCCGCTAcctactgtttctacttccactgttgc }\end{array}$ & $\begin{array}{c}498-521 \\
2382-2407\end{array}$ & AF037448 \\
\hline KSRP & $\begin{array}{l}\text { S-Kpnl: CGGGGTACccgccgcgccatgtcggactacagc } \\
\text { AS-EcoRI: CCGGAATTCCgttcacattcattcgattcattgagc }\end{array}$ & $\begin{array}{c}84-108 \\
2221-2246\end{array}$ & NM_003685 \\
\hline hnRNP-C1 & $\begin{array}{l}\text { S-BamHI: CGCGGATCcggctttgtgagaaaccttaccatc } \\
\text { AS-EcoRI: CCGGAATTCCgtgcttaagagtcatcctccattgg }\end{array}$ & $\begin{array}{c}157-181 \\
1048-1072\end{array}$ & NM_004500 \\
\hline ABBP1 & $\begin{array}{l}\text { S-EcoRI: CCGGAATTccgcgcctcggcctagcatgtcg } \\
\text { AS-Notl: AAGAATGCGGCCGCTAgctgccgcctcagtatggcttgtag }\end{array}$ & $\begin{array}{c}241-263 \\
1241-1265\end{array}$ & NM_031266 \\
\hline hnRNP-A1 & $\begin{array}{l}\text { S-BamHI: CGCGGATcctgccgtcatgtctaagtcag } \\
\text { AS-Notl: AAGAATGCGGCCGCTActggctctcctctcctgctaag }\end{array}$ & $\begin{array}{c}96-117 \\
1081-1102\end{array}$ & NM_002136 \\
\hline ABBP2 & $\begin{array}{l}\text { S-BamHI: CGCGGATCCaacaggacccgggacagaggaaccatg } \\
\text { AS-EcoRI: CCGGAATTCcactctcaatatccttgcagtccattg }\end{array}$ & $\begin{array}{c}216-242 \\
1295-1321\end{array}$ & NM_016306 \\
\hline BAG4 & $\begin{array}{l}\text { S-Kpnl: CGGGGTACCCgcttcagggcagcggatcccatgtc } \\
\text { AS-Notl: AAGAATGCGGCCGCTAgtggaagcctgttactaacttgacc }\end{array}$ & $\begin{array}{c}263-287 \\
1673-1697\end{array}$ & NM_004874 \\
\hline pe6623 & S: AAATTGCATTAGATGATGCC & $6598-6617$ & X04714 \\
\hline pe6639 & S: TGCCAAAATCAACTTTAATG & $6614-6633$ & $\mathrm{X} 04714$ \\
\hline pe6648 & AS: AATTGTATCATATATGTCTG & $6651-6670$ & $\mathrm{X} 04714$ \\
\hline pe6651 & AS: CTGATCAAATTGTATCATATATG & $6655-6677$ & $\mathrm{X} 04714$ \\
\hline pe6655 & AS: TATACTGATCAAATTGTATCAT & $6660-6681$ & $\mathrm{X} 04714$ \\
\hline pe6666 & AS: TATCTTTAATATACTGATC & $333-354$ & $\mathrm{X} 04714$ \\
\hline pe6762 & S: GATGAAATCATTGAAAAATTA & $6735-6755$ & $\mathrm{X} 04714$ \\
\hline pe6802 & S: TCATATCCGTGTAAATTTAG & $6776-6795$ & X04714 \\
\hline pe6845 & S: ACATTTGTTTATTGAAAATATTG & $6815-6837$ & $\mathrm{X} 04714$ \\
\hline $\begin{array}{l}\text { apoB fragment } \\
\text { cloning }\end{array}$ & $\begin{array}{l}\text { S-BamHI: CGCGGATCctgggaaaactcccacagcaag } \\
\text { AS-Notl: GAATGCGGCCGCTAccacattttgaatccaggatgcag }\end{array}$ & $\begin{array}{l}6471-6492 \\
6862-6885\end{array}$ & X04714 \\
\hline apoB PCR & $\begin{array}{l}\text { S: ctgggaaaactcccacagcaag } \\
\text { AS: ccacattttgaatccaggatgcag }\end{array}$ & $\begin{array}{l}6471-6492 \\
6862-6885\end{array}$ & X04714 \\
\hline
\end{tabular}

S, forward sense primer; AS, reverse anti-sense primer; and pe, primer extension.

primers (Table 1). After transformation in bacteria, 20 bacterial clones containing a single molecular copy of the amplified apoB mRNA fragment were randomly selected and were further amplified for DNA plasmid extraction. Each apoB insert sequence in the vector was determined by sequencing analyses from both directions. C-to- $\mathrm{U}$ transition sites and the site mutation rate were determined according to the sequencing data of 20 clones and were summarized as shown in Figure 1A.
For primer extension analyses, primers of either sense or antisense located 4-7 nucleotides (nt) away from a specific detection site were synthesized (Table 1) and purified by polyacrylamide gel electrophoresis. The purified primers were $5^{\prime}$-end labeled by $\left[\gamma^{3}{ }^{32} \mathrm{P}\right]$-ATP $(3000 \mathrm{Ci} / \mathrm{mmol}, 10 \mathrm{mCi} / \mathrm{mL}$, Amersham $)$ with T4 nucleotide kinase at $37^{\circ} \mathrm{C}$ for $40 \mathrm{~min}$. After removal of free $\left[\gamma^{-32} \mathrm{P}\right]-$ ATP by filtration through a G-25 Sepharose column, the ${ }^{32} \mathrm{P}-$ labeled primers were utilized for the determination of apoB 
mRNA editing or hypermutation levels at different sites by thermo cycle primer extension with Thermo Sequenase (USB) as described previously (Patterson et al. 2003). Briefly, total RNA samples were pretreated with DNase RQ1. The first strand cDNA was generated with a random primer using M-MLV reverse transcriptase (Invitrogen) and the PCR was performed for apoB amplification from 6471 to 6886 . The amplicons were then purified using a QIAEX II Gel Extraction kit (Qiagen) for primer extension analyses.

An aliquot of the purified apoB DNA was subjected to thermocycle primer extension for each editing or hypermutation site in the presence of ddGTP for antisense primers or ddCTP for sense primers. The primer extension stops at the cytidine of each detection site because of ddGTP or ddCTP incorporation if the cytidine at the site is not mutated. If the cytidine at the detection site is mutated, the primer extension will continue to the next cytidine available. Thus, the primer extension results in two or more primer extended products observed as additional bands above primer band in gel electrophoresis seperation representing unedited RNA population by the shorter one ( $\mathrm{C}$ at a given site) and edited or hypermutated RNA population by the longer one ( $U$ at the given site) (see Fig. 1). After denaturation, the primer extension products were resolved by electrophoresis in an $8 \%$ polyacrylamide-urea sequencing gel and the ratio of edited to unedited apoB mRNA at a given site was determined by a PhosphorImager.

\section{NAT1 (novel Apobec-1 target no. 1) gene hypermutation analyses}

Primer extension at site 3736 of NAT1 mRNA has been developed as a mean to evaluate APOBEC-1 hypermutation effect on a gene other than apoB in the mouse liver (Yamanaka et al. 1997). NAT1 gene is highly conserved across different species. The hypermutation of NAT1 gene at site 3736 in rat hepatocytes was analyzed by PCR-amplification followed by primer extension as described previously.

\section{Protein interaction analyses using an in vitro translation system}

To determine the protein interaction alteration between APOBEC-1 and ACF, ACF was constructed into a pcDNA3.1 vector (Invitrogene) with a N-terminal FLAG tag. APOBEC-1 mutants were similarly constructed with or without a C-terminal HA tag (Teng et al. 1999). Utilizing these plasmid constructs, various APOBEC-1s and ACF were cotranslated in a T7 TNT quick coupled transcription/translation system (Promega) at $30^{\circ} \mathrm{C}$ for $2 \mathrm{~h}$ in the presence of $\mathrm{L}^{35} \mathrm{~S}$-methionine $(1175 \mathrm{Ci} / \mathrm{mmol}$, PerkinElmer). The protein complexes formed during the in vitro transcription and translation were immunoprecipitated by an antibody against the FLAG tag (Sigma) at room temperature for $30 \mathrm{~min}$ followed by precipitation with Protein G Dynabeads (Invitrogene) according to the manufacturer's instructions. After extensive washing, the immunoprecipitated protein complexes were separated on a $12 \%$ SDS-PAGE denaturing gel and the protein interaction strength was determined by the levels of APOBEC-1 mutants coprecipitated with ACF using a PhosphorImager. As a reference for the comparison, the initial levels of APOBEC-1 mutants involved in the protein interaction with ACF was determined in parallel by resolving an aliquot of each cotranslation mixture before immunoprecipitation in a $12 \%$ SDSPAGE denaturing gel.

\section{ACKNOWLEDGMENTS}

This research was supported by the Intramural Research Programs of the National Institute of Health including the Clinic Center (CC), The National Institute of Allergy and Infectious Diseases (NIAID), The National Heart, Lung, Blood Institute (NHLBI), and National Institute of Diabetes and Digestive and Kidney Diseases.

Received August 3, 2009; accepted February 5, 2010.

\section{REFERENCES}

Anant S, Blanc V, Davidson NO. 2003. Molecular regulation, evolutionary, and functional adaptations associated with C-to-U editing of mammalian apolipoproteinB mRNA. Prog Nucleic Acid Res Mol Biol 75: 1-41.

Blanc V, Kennedy S, Davidson NO. 2003. A novel nuclear localization signal in the auxiliary domain of apobec-1 complementation factor regulates nucleocytoplasmic import and shuttling. J Biol Chem 278: 41198-41204.

Bocharov AV, Huang W, Vishniakova TG, Zaitseva EV, Frolova EG, Rampal P, Bertolotti R. 1995. Glucocorticoids upregulate highaffinity, high-density lipoprotein binding sites in rat hepatocytes. Metabolism 44: 730-738.

Chan L. 1992. Apolipoprotein B, the major protein component of triglyceride-rich and low-density lipoproteins. J Biol Chem 267: 25621-25624.

Chen SH, Habib G, Yang CY, Gu ZW, Lee BR, Weng SA, Silberman SR, Cai SJ, Deslypere JP, Rosseneu M, et al. 1987. Apolipoprotein B-48 is the product of a messenger RNA with an organ-specific in-frame stop codon. Science 238: 363-366.

Chen Z, Eggerman TL, Patterson AP. 2007. ApoB mRNA editing is mediated by a coordinated modulation of multiple apoB mRNA editing enzyme components. Am J Physiol Gastrointest Liver Physiol 292: G53-G65.

Chester A, Somasekaram A, Tzimina M, Jarmuz A, Gisbourne J, O'Keefe R, Scott J, Navaratnam N. 2003. The apolipoprotein B mRNA editing complex performs a multifunctional cycle and suppresses nonsense-mediated decay. EMBO J 22: 3971-3982.

Chester A, Weinreb V, Carter CW Jr, Navaratnam N. 2004. Optimization of apolipoprotein B mRNA editing by APOBEC1 apoenzyme and the role of its auxiliary factor, ACF. RNA 10: 1399-1411.

Funahashi T, Giannoni F, DePaoli AM, Skarosi SF, Davidson NO. 1995. Tissue-specific, developmental and nutritional regulation of the gene encoding the catalytic subunit of the rat apolipoprotein $B$ mRNA editing enzyme: Functional role in the modulation of apoB mRNA editing. J Lipid Res 36: 414-428.

Hersberger M, Innerarity TL. 1998. Two efficiency elements flanking the editing site of cytidine 6666 in the apolipoprotein B mRNA support mooring-dependent editing. J Biol Chem 273: 94359442.

Hersberger M, Patarroyo-White S, Qian X, Arnold KS, Rohrer L, Balestra ME, Innerarity TL. 2003. Regulatable liver expression of the rabbit apolipoprotein B mRNA-editing enzyme catalytic polypeptide 1 (APOBEC-1) in mice lacking endogenous APOBEC-1 leads to aberrant hyperediting. Biochem J 369: 255-262.

Hughes SD, Rouy D, Navaratnam N, Scott J, Rubin EM. 1996. Gene transfer of cytidine deaminase apoBEC-1 lowers lipoprotein(a) in transgenic mice and induces apolipoprotein B editing in rabbits. Hum Gene Ther 7: 39-49.

Innerarity TL, Mahley RW. 1978. Enhanced binding by cultured human fibroblasts of apo-E-containing lipoproteins as compared with low-density lipoproteins. Biochemistry 17: 1440-1447.

Innerarity TL, Boren J, Yamanaka S, Olofsson SO. 1996. Biosynthesis of apolipoprotein B48-containing lipoproteins. Regulation by novel post-transcriptional mechanisms. J Biol Chem 271: 23532356. 
Kozarsky KF, Bonen DK, Giannoni F, Funahashi T, Wilson JM, Davidson NO. 1996. Hepatic expression of the catalytic subunit of the apolipoprotein B mRNA editing enzyme (apobec-1) ameliorates hypercholesterolemia in LDL receptor-deficient rabbits. Hum Gene Ther 7: 943-957.

Maris C, Masse J, Chester A, Navaratnam N, Allain FH. 2005. NMR structure of the apoB mRNA stem-loop and its interaction with the C-to-U editing APOBEC1 complementary factor. RNA 11: 173-186.

Patterson AP, Chen Z, Rubin DC, Moucadel V, Iovanna JL, Brewer HB Jr, Eggerman TL. 2003. Developmental regulation of apolipoprotein B mRNA editing is an autonomous function of small intestine involving homeobox gene Cdx1. J Biol Chem 278: 76007606.

Pitas RE, Innerarity TL, Mahley RW. 1980. Cell surface receptor binding of phospholipid. protein complexes containing different ratios of receptor-active and -inactive E apoprotein. J Biol Chem 255: 5454-5460.

Powell LM, Wallis SC, Pease RJ, Edwards YH, Knott TJ, Scott J. 1987. A novel form of tissue-specific RNA processing produces apolipoprotein-B48 in intestine. Cell 50: 831-840.

Qian X, Balestra ME, Yamanaka S, Boren J, Lee I, Innerarity TL. 1998. Low expression of the apolipoprotein B mRNA-editing transgene in mice reduces LDL levels but does not cause liver dysplasia or tumors. Arterioscler Thromb Vasc Biol 18: 1013-1020.

Siddiqui JF, Van Mater D, Sowden MP, Smith HC. 1999. Disproportionate relationship between APOBEC-1 expression and apolipoprotein B mRNA editing activity. Exp Cell Res 252: 154-164.

Smedsrod B, Pertoft H, Eggertsen G, Sundstrom C. 1985. Functional and morphological characterization of cultures of Kupffer cells and liver endothelial cells prepared by means of density separation in Percoll, and selective substrate adherence. Cell Tissue Res 241: 639-649.

Sowden M, Hamm JK, Smith HC. 1996a. Overexpression of APOBEC-1 results in mooring sequence-dependent promiscuous RNA editing. J Biol Chem 271: 3011-3017.

Sowden M, Hamm JK, Spinelli S, Smith HC. 1996b. Determinants involved in regulating the proportion of edited apolipoprotein $\mathrm{B}$ RNAs. RNA 2: 274-288.
Sowden MP, Eagleton MJ, Smith HC. 1998. Apolipoprotein B RNA sequence $3^{\prime}$ of the mooring sequence and cellular sources of auxiliary factors determine the location and extent of promiscuous editing. Nucleic Acids Res 26: 1644-1652.

Teng B, Blumenthal S, Forte T, Navaratnam N, Scott J, Gotto AM Jr, Chan L. 1994. Adenovirus-mediated gene transfer of rat apolipoprotein $\mathrm{B}$ mRNA-editing protein in mice virtually eliminates apolipoprotein B-100 and normal low density lipoprotein production. J Biol Chem 269: 29395-29404.

Teng B, Ishida B, Forte TM, Blumenthal S, Song LZ, Gotto AM Jr, Chan L. 1997. Effective lowering of plasma, LDL, and esterified cholesterol in LDL receptor-knockout mice by adenovirus-mediated gene delivery of ApoB mRNA editing enzyme (Apobec1). Arterioscler Thromb Vasc Biol 17: 889-897.

Teng BB, Ochsner S, Zhang Q, Soman KV, Lau PP, Chan L. 1999. Mutational analysis of apolipoprotein B mRNA editing enzyme (APOBEC1). structure-function relationships of RNA editing and dimerization. J Lipid Res 40: 623-635.

Yamanaka S, Poksay KS, Balestra ME, Zeng GQ, Innerarity TL. 1994. Cloning and mutagenesis of the rabbit ApoB mRNA editing protein. A zinc motif is essential for catalytic activity, and noncatalytic auxiliary factor(s) of the editing complex are widely distributed. J Biol Chem 269: 21725-21734.

Yamanaka S, Balestra ME, Ferrell LD, Fan J, Arnold KS, Taylor S, Taylor JM, Innerarity TL. 1995. Apolipoprotein B mRNA-editing protein induces hepatocellular carcinoma and dysplasia in transgenic animals. Proc Natl Acad Sci 92: 8483-8487.

Yamanaka S, Poksay KS, Driscoll DM, Innerarity TL. 1996. Hyperediting of multiple cytidines of apolipoprotein B mRNA by APOBEC-1 requires auxiliary protein(s) but not a mooring sequence motif. J Biol Chem 271: 11506-11510.

Yamanaka S, Poksay KS, Arnold KS, Innerarity TL. 1997. A novel translational repressor mRNA is edited extensively in livers containing tumors caused by the transgene expression of the apoB mRNA-editing enzyme. Genes \& Dev 11: 321-333.

Young SG. 1990. Recent progress in understanding apolipoprotein B. Circulation 82: 1574-1594. 

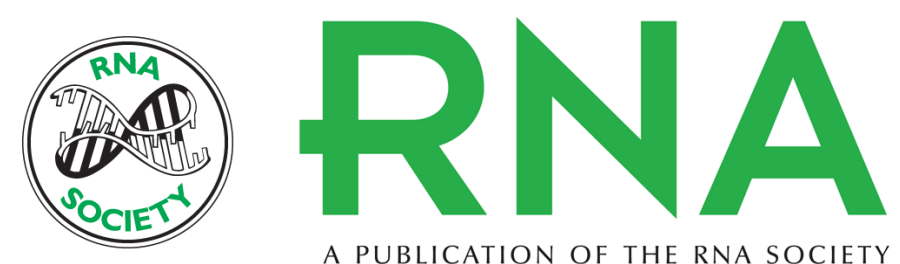

A PUBLICATION OF THE RNA SOCIETY

\section{Hypermutation induced by APOBEC-1 overexpression can be eliminated}

Zhigang Chen, Thomas L. Eggerman, Alexander V. Bocharov, et al.

RNA 2010 16: 1040-1052 originally published online March 26, 2010

Access the most recent version at doi:10.1261/rna.1863010

$\begin{array}{ll}\text { References } & \begin{array}{l}\text { This article cites } 33 \text { articles, } 22 \text { of which can be accessed free at: } \\ \text { http://rnajournal.cshlp.org/content/16/5/1040.full.html\#ref-list-1 }\end{array}\end{array}$

License

Email Alerting Receive free email alerts when new articles cite this article - sign up in the box at the Service top right corner of the article or click here. 